\title{
Photodamage in deep tissue two-photon optical biopsy of human skin
}

\author{
Luca Dalbosco ${ }^{1}$, Giulia Zanini ${ }^{2}$, Elvira D'Amato ${ }^{2}$, Francesco Tessarolo $^{1,3}$, Sebastiana Boi ${ }^{4}$, Paolo Bauer ${ }^{5}$, Albrecht \\ Haase $^{2, *}$, and Renzo Antolini ${ }^{2}$ \\ ${ }^{1}$ BIOtech center and Department of Industrial Engineering, University of Trento, via delle Regole 101, 38123 Mattarello, Italy \\ ${ }^{2}$ Department of Physics, University of Trento, via Sommarive 14, 38123 Povo, Italy \\ ${ }^{3}$ Healthcare Research and Innovation Program (IRCS), Bruno Kessler Foundation, 38122 Trento, Italy \\ ${ }^{4}$ Department of Pathology, APSS, S. Chiara Hospital, Largo Medaglie d'Oro 9, 38122 Trento, Italy \\ ${ }^{5}$ Department of Dermatology, APSS, S. Chiara Hospital, Largo Medaglie d'Oro 9, 38122 Trento, Italy
}

Received zzz, revised zzz, accepted zzz

Published online $z z z$

Key words: Biomedical imaging, Nonlinear Microscopy, Skin imaging, Photodamage, Multiphoton processes

\begin{abstract}
Photodamage, induced by femtosecond laser radiation, was studied in thick samples of human skin tissue (healthy skin and neoplastic lesions). Photobleaching, photoionization, and thermomechanical damage effects were characterized comparatively. The laser power dependence of the damage rates allowed to connect macroscopic effects to underlying molecular processes. Optical effects were correlated to histopathological changes. Tissue alterations were found only from thermomechanical cavitation and limited to superficial layers of the epidermis. From the depth-dependencies of all damage thresholds a depth-dependent power-compensation scheme was defined which allows for damage-free deep tissue optical biopsy.
\end{abstract}

\section{Introduction}

Skin cancer and, in particular, its most aggressive type, the melanoma, shows an alarming increase, with the number of cases doubling every 10-20 years [1]. In the European Union in 2012 the mortality rate was 22 (cases per million) [2]. This underlines the importance of the development of techniques that on one hand allow for an early diagnosis and on the other hand permit a broad screening of the public, which requires fast, cost-effective, and noninvasive applicability.

So far, the clinical diagnostic gold standard is the biopsy which is analysed histologically, a technique that doesn't fulfil any of the previous criteria.
Damage induced luminescence pattern for different excitation powers and a corresponding threshold analysis.

Different imaging modalities are currently evaluated in clinical trials to overcome this problem. The most advanced high resolution, non-invasive methods are laser scanning confocal microscopy (LSCM) [3], optical coherence tomography (OCT) [4], and multiphoton microscopy (MPM) [5]. LSCM and OCT both rely on reflection and scattering index modulations as contrast generating mechanism and provide high resolution morphological information. MPM provides also chemical information via the intrinsic signal of different endogenous fluorophores and information on the fibrillar structure via the second harmonic generation signal of skin's intrinsic compounds with higher order susceptibility [6].

\footnotetext{
* Corresponding author: e-mail albrecht.haase@unitn.it,Phone: +43 046128 3650, Fax: +43 0461283659
} 
Author, Author, and Author: Short title

MPM offers further favourable properties; thanks to the signal's nonlinear excitation power dependence, the absorption volume is limited to the immediate vicinity of the focus [7]. This implies an intrinsically high spatial resolution, an increased penetration depth, and also damaging effects are confined to the focus and therefore strongly reduced $[5,8]$. But in turn, to excite these nonlinear transitions, the necessary peak powers of the laser source need to be on the order of $\mathrm{kW}$. However, thanks to femtosecond pulsed lasers, these peak powers can be achieved with average powers of the order of $\mathrm{mW}$. Still there is the need to study possible damaging effects this method could be causing.

Previous studies on ultra-short pulsed laser photodamage concentrated mostly on cell cultures and investigated the dependence on peak intensities $[9,10]$, mean power $[11,12]$, and pulse length $[10,13,14]$. The dependences on mean power [15-17], pulse length [18], and repetition rate [16] were studied also in tissue slices. For the standard Ti:Sa laser source producing $100 \mathrm{fs}$ pulses at a $80 \mathrm{MHz}$ repetition rate, damage thresholds in cell cultures were estimated to be of the order of $0.8 \mathrm{~kW}$ peak power, corresponding to an average power of $10 \mathrm{~mW}$ at $800 \mathrm{~nm}$ wavelength [9].

The monitored damages suggest three underlying mechanisms. The first is a photochemical damage, where, after two-photon excitation, intracellular coenzymes like $\mathrm{NAD}(\mathrm{P}) \mathrm{H}$ act as photosensitizers, creating reactive oxygen species. Cells respond with reduced cell vitality [12] and cell death via apoptosis [19]. The effect was found to be similar to the damage caused by single UV photon absorption $[9,15]$. An immediate symptom which can be observed under the microscope is photobleaching, since the photochemical modification of $\mathrm{NAD}(\mathrm{P}) \mathrm{H}$ extinguishes the autofluorescence of the excited molecules. Recent studies show increased carcinogenesis in cells exposed to fs infrared radiation with respect to UV exposure [20]. A second damaging effect is of thermo-mechanical origin. Single near-infrared (NIR) photon absorption (e.g. in melanin) causes local heating, water evaporation, and cavitation. Cells and tissue are disrupted mechanically by explosive vaporization. The effect can be observed immediately due to bubble formation in melanin-rich areas [16,21]. A third effect, caused by the strong electric fields at the peak power, is photoionization and plasma formation. This effect was reported to be accompanied by the onset of strong luminescence from newly formed compounds [9,22].

Most of these damage studies were performed in various cell cultures, some of them analyse single effects also in skin tissue [15-17]. Still missing is a comprehensive study of photodamage in thick skin tissue under varying imaging parameters.

In this work, the different damage effects were identified and quantified, not only as a function of power and exposure time, but also depending on the imaging depth within the tissue. This allows to determine depth-dependent damage thresholds, from which damage-free protocols for depth-dependent power compensation in 3D deep tissue optical biopsy can be defined.

\section{Experimental}

\subsection{Optical setup}

The optical setup was a two-photon microscope (Ultima IV, Prairie Technologies) combined with an ultra-short pulsed laser (Mai Tai Deep See HP, Spectra-Physics). The laser was tuned to $800 \mathrm{~nm}$ for autofluorescence imaging, and to $990 \mathrm{~nm}$ for second harmonic generation (SHG). A Pockels cell controlled the laser beam power which was varied between 2 and $100 \mathrm{~mW}$ at the sample surface. Galvo-mirrors were used to scan the samples. The excitation light was strongly focused onto the sample by a water immersion objective (20×, NA 1.0, Olympus). Fluorescence was collected in an epi-geometry, separated from the backscattered excitation light by a dichroic beamsplitter (cutting off at $660 \mathrm{~nm}$ ), filtered by a $70 \mathrm{~nm}$ bandpass filter centred around $525 \mathrm{~nm}$ (Chroma Technology), and detected by photomultiplier tubes (Hamamatsu Photonics). A second channel with a detection window of $607 \pm 23 \mathrm{~nm}$ was used in addition to estimate the luminescence bandwidth.

\subsection{Patients and biopsies}

Creating tissue damages intentionally, these experiments could not be performed in vivo because of ethical and safety reasons. Therefore, tissue samples were collected from regular biopsies of a total of 8 patients, median age 57, range: 31-91, male:female 3:5, 100\% Caucasian skin type. The study was approved by the local Ethical committee (Protocol ID21419631/2011, Ethical committee for clinical trials, Azienda Provinciale per i Servizi Sanitari di Trento). All patients were recruited at the Department of Dermatology of S. Chiara Hospital and gave written informed consent.

Biopsies were representative for control skin, skin lesions (melanocytic nevi, basal cell carcinomas (BCCs), and melanomas) and perilesional skin. Three biopsies were excised from head and neck, three from trunk and two from upper limbs. From the clinically harvested biopsies, three samples of healthy skin, one melanocytic nevus, three basal cell carcinomas, and one melanoma were considered for two-photon microscopy analysis.

\subsection{Sample treatment}

All tissue specimens were fixed in buffered formalin immediately after excision. Fixed tissue was used to prevent degradation during subsequent optical and histological analysis. Exemplary tests of freshly excised tissues were 
showing comparable results. Briefly, after complete fixation (at least $12 \mathrm{~h}$ ), samples were washed in phosphate buffered saline solution (PBS) and mounted onto a custom-made holder. To minimize morphological surface variability, samples were covered with a cover slip which was gently pressed against the tissue by springs. After studies via two-photon fluorescence microscopy and second harmonic generation, all samples were subsequently paraffin embedded for specific histochemistry and immuno-histochemistry analysis.

Table 1 Imaging protocols and samples.

\begin{tabular}{|c|c|c|c|c|c|c|c|}
\hline $\begin{array}{l}\text { Imaging } \\
\text { protocol }\end{array}$ & $\begin{array}{l}\text { Laser } \\
\text { power* }\end{array}$ & $\begin{array}{l}\text { Repetitions, } \\
\text { Frame rate }\end{array}$ & $\begin{array}{l}\text { Imaging } \\
\text { depth** }\end{array}$ & $\begin{array}{l}\text { Pixel } \\
\text { exposure } \\
\text { time }\end{array}$ & $\begin{array}{l}\text { Pixel } \\
\text { resolution }\end{array}$ & $\begin{array}{l}\text { Exposed } \\
\text { area }\end{array}$ & $\begin{array}{l}\text { Sample type } \\
\text { (quantity) }\end{array}$ \\
\hline $\begin{array}{l}\text { Low frame rate } \\
\text { photobleaching }\end{array}$ & $10-83 \mathrm{~mW}$ & $\begin{array}{l}200 \text { times, } \\
0.6 \mathrm{~Hz}\end{array}$ & $30,60,90 \mu \mathrm{m}$ & $5.2 \mu \mathrm{s}$ & $512 \times 512$ & $352 \times 352 \mu \mathrm{m}^{2}$ & $\begin{array}{l}\text { Healthy skin (3) } \\
\text { Melanoma (1) }\end{array}$ \\
\hline $\begin{array}{l}\text { High frame rate } \\
\text { photobleaching }\end{array}$ & $10-83 \mathrm{~mW}$ & $\begin{array}{l}200 \text { times, } \\
13 \mathrm{~Hz}\end{array}$ & $30 \mu \mathrm{m}$ & $5.2 \mu \mathrm{s}$ & $100 \times 100$ & $70 \times 70 \mu \mathrm{m}^{2}$ & Healthy skin (2) \\
\hline $\begin{array}{l}\text { Photoionization and } \\
\text { thermomechanical } \\
\text { damage }\end{array}$ & $21-81 \mathrm{~mW}$ & $\begin{array}{l}10 \text { times, } \\
0.1 \mathrm{~Hz}\end{array}$ & $30,80,130 \mu \mathrm{m}$ & $50 \mu \mathrm{s}$ & $512 \times 512$ & $\begin{array}{l}12 \text { ROIs of } 65 \times 65 \mu^{2} \\
\text { in } 352 \times 352 \mu \mathrm{m}^{2} \mathrm{FOV}\end{array}$ & $\begin{array}{l}\text { Healthy skin (1) } \\
\text { Melanoma (1) }\end{array}$ \\
\hline $\begin{array}{l}\text { Thermomechanical } \\
\text { damage- full power }\end{array}$ & $100 \mathrm{~mW}$ & $\begin{array}{l}10 \text { times, } \\
0.1 \mathrm{~Hz}\end{array}$ & $\begin{array}{l}\text { between } 30 \\
\text { and } 40 \mu \mathrm{m}\end{array}$ & $50 \mu \mathrm{s}$ & $512 \times 512$ & $\begin{array}{l}36 \text { ROIs of } 52 \times 52 \mu \mathrm{m}^{2} \\
\text { in } 528 \times 528 \mu \mathrm{m}^{2} \mathrm{FOV}\end{array}$ & $\begin{array}{l}\text { BCC (2) } \\
\text { Healthy skin (2) }\end{array}$ \\
\hline $\begin{array}{l}\text { Thermomechanical } \\
\text { damage- variable } \\
\text { power }\end{array}$ & $26-81 \mathrm{~mW}$ & $\begin{array}{l}7 \text { times, } \\
0.1 \mathrm{~Hz}\end{array}$ & $\begin{array}{l}\text { between } 30 \\
\text { and } 40 \mu \mathrm{m}\end{array}$ & $50 \mu \mathrm{s}$ & $512 \times 512$ & $\begin{array}{l}5 \text { ROIs of } 44 \times 300 \mu \mathrm{m}^{2} \\
\text { in } 352 \times 352 \mu \mathrm{m}^{2} \mathrm{FOV}\end{array}$ & $\begin{array}{l}\text { Melanocytic } \\
\text { nevus (1) }\end{array}$ \\
\hline $\begin{array}{l}\text { Power- } \\
\text { compensation }\end{array}$ & $3-30 \mathrm{~mW}$ & - & $\begin{array}{l}0 \text { to } 200 \mu \mathrm{m} \\
\text { in } 5 \mu \mathrm{m} \text { steps }\end{array}$ & $50 \mu \mathrm{s}$ & $512 \times 512$ & $352 \times 352 \mu \mathrm{m}^{2}$ & Healthy skin (1) \\
\hline
\end{tabular}

* after the objective, ${ }^{* *}$ from the uppermost stratum corneum, ROI: region of interest, FOV: field of view

\subsection{Imaging protocols}

In order to obtain absolute depth-dependent information, the sample surface was carefully assessed in every experiment defining it as the first imaging plane showing anucleated keratinocytes of the uppermost stratum corneum, excluding exfoliated cells, in more than one half of the field of view.

Different imaging protocols were applied to each tissue sample, but varying the exposed volume to avoid superimposition of damaging effects.

Six different experimental protocols were defined in order to induce and quantify NIR fs-laser photodamage and to optimize deep tissue imaging.

Namely, two protocols were designed to assess photobleaching at high and low frame rate respectively; one protocol evaluated photoionization and thermo-mechanical damage; two protocols aimed at visualizing thermomechanical damage in comparison with histology. Finally, a specific protocol was defined to compensate scat- tering loss in deep tissue imaging down to $200 \mu \mathrm{m}$. Protocols details and acquisition parameters are summarized in Table 1.

Photobleaching protocols consisted in repeated laser application with short exposures times. A sample area of $352 \times 352 \mu \mathrm{m}^{2}$ was irradiated with $5.2 \mu \mathrm{s}$ pixel exposure time using $512 \times 512$ pixels resolution at a frame rate of 0.6 Hz. This procedure was repeated for six different laser powers ranging from 10 to $83 \mathrm{~mW}$ at the sample, each time at unexposed and morphologically uniform regions. A second protocol used a frame rate of $13 \mathrm{~Hz}$, reducing the field of view to $70 \times 70 \mu \mathrm{m}^{2}$, resolved in $100 \times 100$ pixels. In all these experiments a control of the initial fluorescence intensity dependence on the laser power was performed, based on the first image of each series.

In the photoionization protocol, the induced luminescence enhancement was studied depending on power and depth. To determine power thresholds the same field of view was subdivided into a pattern of 12 quadratic regions of interest (ROIs) to which expose was limited $\left(65 \times 65 \mu \mathrm{m}^{2}\right)$ with a spacing comparable to their size, so that about half of 
Author, Author, and Author: Short title

the total area was illuminated. Each ROI was irradiated with a specific power between 21 and $81 \mathrm{~mW}$. To study cumulative effects, exposures were repeated 10 times, with a frame rate of $0.1 \mathrm{~Hz}$ and $50 \mu \mathrm{s}$ pixel exposure time. In between high power exposures, samples were imaged at a minimum power of $2 \mathrm{~mW}$, allowing to compare damage progression without contributing to it. Not only exposed regions were studied, but also the unexposed zones in between. All samples subjected to the above described protocol were also analysed for thermomechanical damages.

To evaluate thermomechanical damage, two additional protocols with different exposure parameters were designed. One protocol used a grid of 36 ROIs (each $52 \times 52$ $\mu \mathrm{m}^{2}$ ). Each ROI was exposed to the same laser power of $100 \mathrm{~mW}, 50 \mu$ s pixel exposure time, 10 repetitions were performed with a frame rate of $0.1 \mathrm{~Hz}$. A second protocol consisted in exposing 7 times the same sample area with stepwise increasing powers from 26 to $81 \mathrm{~mW}$.

To simplify the recognition of the treated zones for the histological analysis, the exposure was extended over several neighbouring regions to an overall area of $2 \mathrm{~mm}^{2}$.

In the power compensation protocol, an optimal modulation of the excitation laser power had to achieved to compensate for the exponential scattering loss with increasing imaging depth. During the acquisition of an image stack, the power was varied via adapting the voltage at a Pockels cell following the equation:

$$
P(z)=P_{0}+\left(P_{f}-P_{0}\right) \frac{{\frac{}{z-z_{0}}}^{\frac{z_{f}-z_{0}}{d}}-1}{e^{\frac{z}{d}}}
$$

where $P_{0}$ is the power at the image stack's upper limit $z_{0}$, and $P_{\mathrm{f}}$ the power at the final imaging depth $z_{\mathrm{f}}$. The parameter $d$ defines a characteristic compensation length, which was determined by optimizing the fluorescence depth profile. A reasonable optimization parameter is the average fluorescence intensity of the single images, which should be kept constant with increasing imaging depth. Autofluorescence images were acquired from the surface $\left(z_{0}\right)$ down to $200 \mu \mathrm{m}\left(z_{\mathrm{f}}\right)$ every $5 \mu \mathrm{m} . P_{\mathrm{o}}$ and $P_{\mathrm{f}}$ were chosen to optimize and balance the overall fluorescence signal at both ends of the image stack. The best exponential compensation length $d$ was determined empirically by varying its value from 0 (for no compensation) to $d>>1$ (for linear compensation).

\subsection{Image analysis}

The acquired images were directly imported into Matlab (Mathworks) where all post-processing and image analysis were performed. After a general background subtraction and intensity normalization, within the single regions of interest (ROIs), the autofluorescence signal was aver- aged over the respective area for every frame. This average fluorescence intensity was then analysed as a function of time, imaging depth, laser power, and other experimental parameters.

To study photobleaching, a double-exponential of the form

$$
\bar{F}(t)=A_{0}+A_{\text {fast }} e^{-k_{\text {fast }} t}+A_{\text {slow }} e^{-k_{\text {slow }} t}
$$

was fitted to the average intensity decay curves.

$k_{\text {fast }}$ and $k_{\text {slow }}$ are the rates of the two decay processes that were observed in the experiments. The underlying decay processes could be determined by evaluating the powerdependence of these decay rates via linear fits to the loglog plots of decay rate versus laser power (Figure 2). Variances were obtained from statistics over independently exposed ROIs.

In the photoionization studies, the area exhibiting a luminescence increase (Figure 3) was determined via a pixelby-pixel evaluation whether the signal exceeded the initial intensity by more than three standard deviations.

The image stacks resulting from the power compensated protocol were reconstructed in 3D using linear interpolation along the optical axis (implemented in Matlab) and direct volume rendering (Amira, VSG).

\subsection{Histological analysis protocol}

A histological analysis was performed on all exposed tissue samples to elicit any histopathologically relevant modification due to the exposure. Immediately after the execution of the imaging protocol, treated areas were marked with black ink and samples were paraffin embedded and serially sectioned transversally to the skin surface. Usually 20 consecutive $5 \mu \mathrm{m}$ thick slices crossing the exposed areas were prepared and stained with HematoxylinEosin (HE) and Periodic acid-Schiff (PAS). Immunohistochemistry for E-cadherin antibodies was performed on consecutive sections to highlight the quality of intercellular keratinocyte junctions in the epidermis close to the irradiated area. All tissue sections were microscopically examined by a senior histopathologist with long expertise in the diagnosis of skin lesions.

\section{Results}

Repeated irradiations of both healthy skin and neoplastic lesion samples showed that the mean intensity of the reemitted light followed first a continuous exponential decay, which results from photobleaching processes of the endogenous fluorophores. After a certain threshold, the signal abruptly increases, surpassing the initial fluorescence multiple times. Finally, at high powers, the signal decreases again because of cavitation and tissue ablation in the imaging plane. 


\subsection{Photobleaching}

To identify the involved photobleaching mechanisms, from the average fluorescence decay curves (Figure 1) bleaching rates were deduced and are shown in log-log plots as a function of the exciting laser power (Figure 2). The gradients of the linear fits reflect the power-law de-
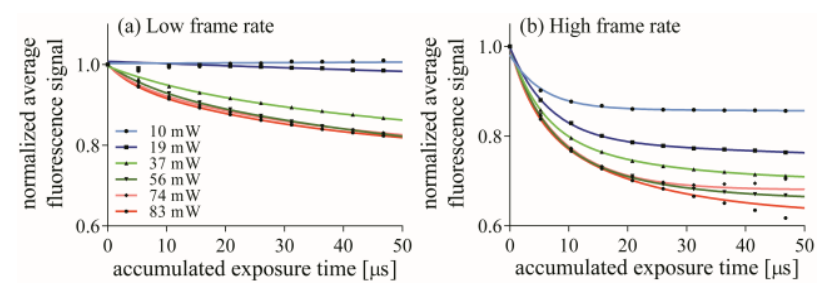

pendences on the laser power and allow to obtain information on the order of the absorption processes that are causing the bleaching.

Figure 1 Fluorescence decay curves for the low frame rate (a) and the high frame rate experiment in (b). The different curves correspond to different excitation powers as shown in the legend.

For slow frame rate exposure $(0.6 \mathrm{~Hz})$, bleaching rates of the order of $10^{-3}-10^{-1} \mu \mathrm{s}^{-1}$ were obtained, which follow power laws with exponents between $2.0 \pm 0.2$ and $4.1 \pm$ 0.4 (Figure 2(a,b)) depending on samples, and tissue regions. Rate distributions were comparable in healthy tissue and melanoma. This indicates that the photobleaching processes included the absorption of two and more photons.

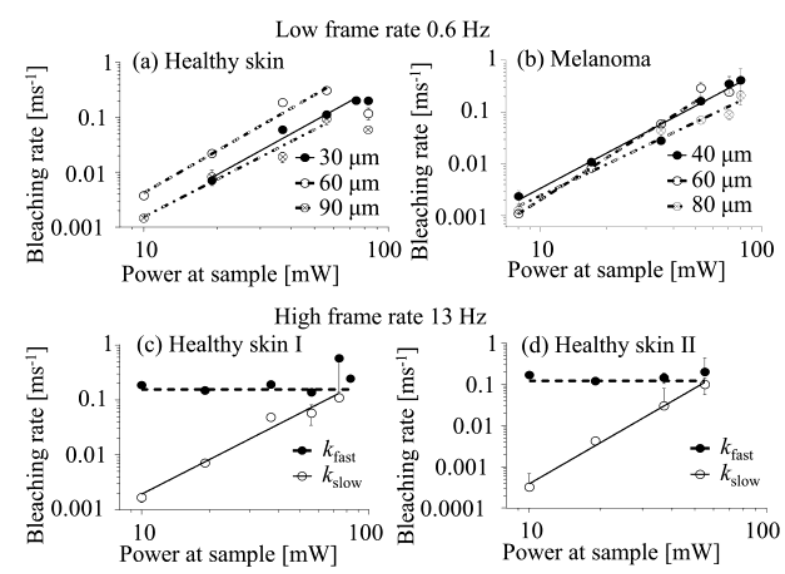

Figure 2 Photobleaching rates as a function of exciting laser power. Rates obtained by low frame rate exposure in a healthy skin sample (a) and in melanoma (b). To fast frame rate data, double exponential decays has to be fitted (c,d). The slow rates follow a power-law comparable to the results in $(a, b)$, the fast decay is power-independent.

For faster frame rates of $13 \mathrm{~Hz}$, double exponential fits gave long-term decay constants with power law exponents between $1.7 \pm 0.1$ and $3.3 \pm 0.2$, confirming the effects at low frame rate exposure. But additionally there is a short- term exponential decay without any power dependence of $0.14 \pm 0.03 \mu \mathrm{s}^{-1}$ for different samples (Figure 2(c,d)).

With every bleaching experiment also the power dependence of the initial fluorescence intensity was tested, resulting in exponents between 1.6 and $1.9( \pm 0.1)$, which confirms the dominance of two-photon absorption.

\subsection{Photoionization}

In both types of tissue, healthy skin and melanoma, an increase in the luminescence intensity after repeated illumination at high powers could be observed. All quantitative measurements presented here were performed at $525 \mathrm{~nm}$, but simultaneous monitoring of the signal at $607 \mathrm{~nm}$ showed the same enhancement pattern. The dependence of this effect on the laser power, the exposure repetition time, and the imaging depth are summarized in Figure 3.

At an imaging depth of $30 \mu \mathrm{m}$ (Figure 3(a)), at low laser powers $(\leq 54 \mathrm{~mW}$ for healthy skin, $\leq 37 \mathrm{~mW}$ for melanoma), the average signal intensity from the exposed regions increased monotonically with the number of exposures to up to 6 times the initial value. The data show that the effect's onset is shifted to fewer repetitions with increasing laser power. In melanoma, it starts even faster than in healthy skin.

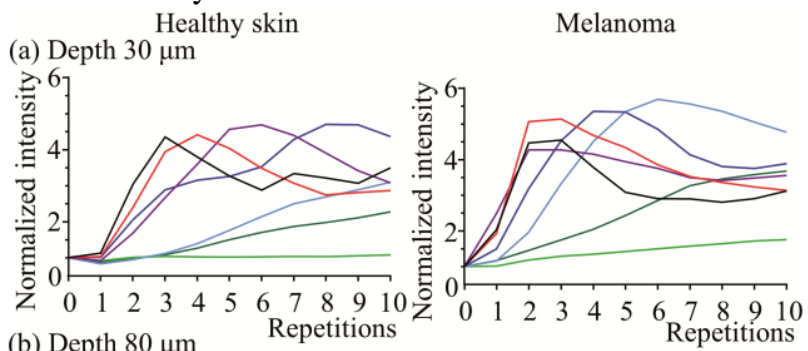

(b) Depth $80 \mu \mathrm{m}$
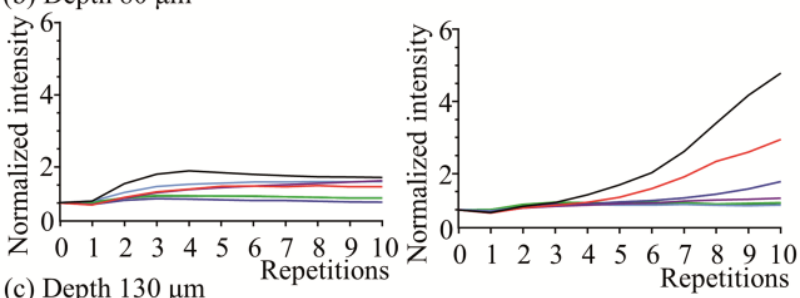

(c) Depth $130 \mu \mathrm{m}$
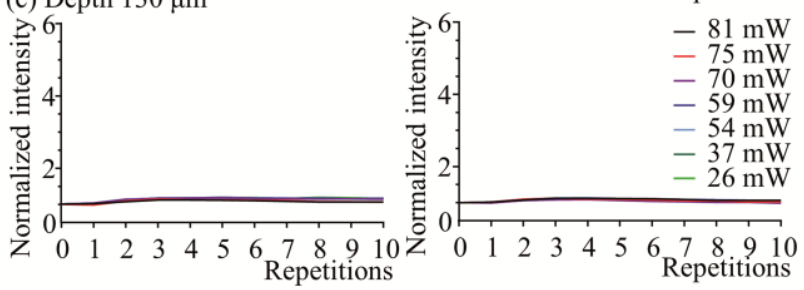

Figure 3 Luminescence intensity curves as a function of the number of exposures. (a-c) Curves for different laser powers in healthy skin at 30,80 , and $130 \mu \mathrm{m}$ imaging depth. (d-f) Same curves for melanoma. 
To determine the exact onset of the effects, the relative area which shows an increased luminescence intensity is evaluated after 7 and more repetitions at $30 \mu \mathrm{m}$ imaging depth and plotted as a function of laser power (Figure 4).
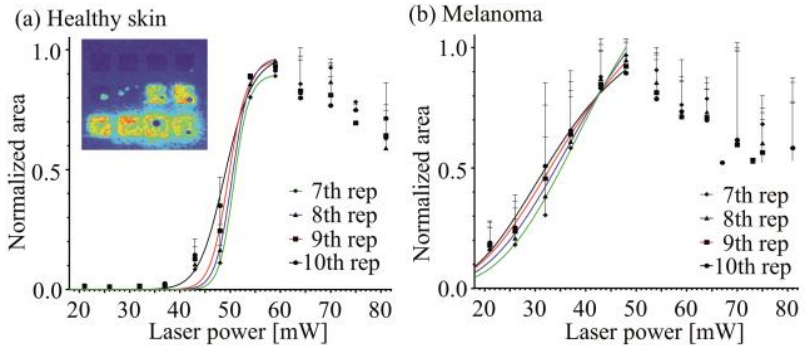

Figure 4 The relative area of exposed regions showing luminescence increase is plotted as a function of laser power in healthy skin (a) and melanoma (b). Inset: Example of an exposed sample irradiated at 12 ROIs $\left(65 \times 65 \mu \mathrm{m}^{2}\right)$ with powers from 21 to 81 $\mathrm{mW}$. Different curves show results after different number of exposures. Error bars represent the standard deviation.

This reveals a much stronger difference between the two sample types. In healthy skin the onset is very sudden and allows to determine a rather precise threshold. While at 37 $\mathrm{mW}$ there is no sign of damage, at $54 \mathrm{~mW}$ almost the complete exposed region is affected. In melanoma, instead, the effect sets in already at about $20 \mathrm{~mW}$ and does not show a clear power threshold behaviour, but rather a linear increase until about $50 \mathrm{~mW}$, where the whole exposed area is affected.

Repeating the experiment at an imaging depth of $80 \mu \mathrm{m}$ (Figure 3(b)) the effect was strongly reduced. Differences between the sample types are very evident, in healthy skin the effect sets in at $54 \mathrm{~mW}$, but the average luminescence intensity doesn't exceed two times the initial value, even for the highest laser powers. In melanoma, instead, the onset is at $59 \mathrm{~mW}$ and shifted towards higher repetitions, but in the case of highest laser power a 5-fold increase of the luminescence intensity can be observed.

At imaging depths of $130 \mu \mathrm{m}$ no signs of luminescence intensity increase could be observed even for the highest laser power of $81 \mathrm{~mW}$ at the sample surface (Figure 3(c)).

\subsection{Thermomechanical damage}

For higher laser powers ( $>54 \mathrm{~mW}$ for healthy skin, $>37 \mathrm{~mW}$ for melanoma) the luminescence increase, described in the previous paragraph, saturates at about 4-5 times the initial intensity and then drops again. An analysis of the single images shows that this drop is due to cavitation in the exposed regions. Bubbles are formed, within which the fluorescence completely vanishes (Figure 4(a) inset). The effect can be explained by the onset of thermomechanical damages due to single photon absorption in melanin driving local tissue heating. Explosive evaporation forms cavities and finally completely ablates the tissue. So the reversal in the luminescence dynamics is due to this morphologically destructive process removing tissue from the imaging volume. The threshold for the thermal damage after five exposures can be estimated from the power corresponding to the peak luminescence intensity, which was $60 \mathrm{~mW}$ in healthy skin and $50 \mathrm{~mW}$ in melanoma. Important to notice is the fact that the onset of thermomechanical damage was always at higher powers or at higher number of repetitions than the luminescence increase.

\subsection{Histological analysis}

A histological analysis allowed to study which structural changes correspond to the changes in the optical signal. None of the tissue samples exhibiting photobleaching or luminescence increase showed any structural alterations. The thermomechanical damage instead caused severe tissue deformations, but in all experiments these were limited to superficial layers above $50 \mu \mathrm{m}$, in agreement with the optical signals for cavitation.
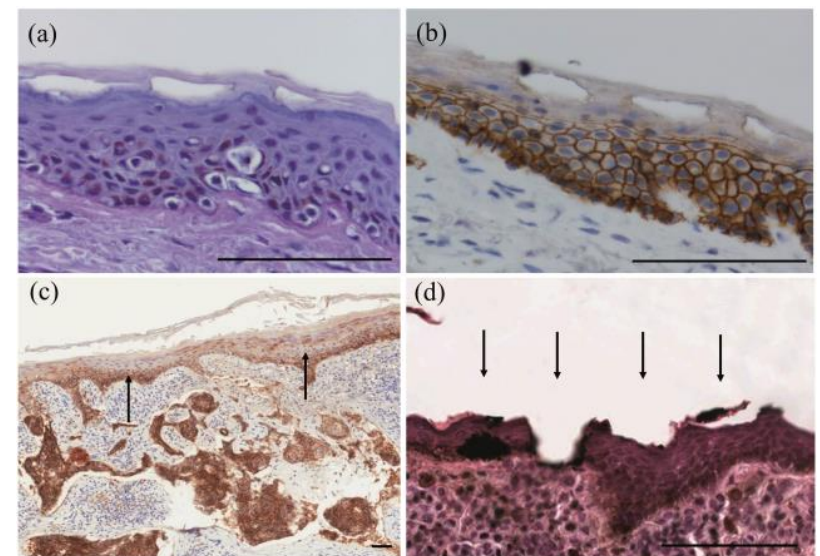

(d)

Figure 5 (a) HE stained perilesional skin sample showing cavities in the stratum corneum of the same dimensions as the exposure mask (arrows), additional PAS staining proves the integrity of the stratum basale. (b) The same sample with E-cadherin staining shows a homogeneous antibody distribution in all epidermal layers. (c) A BBC sample, where the stratum corneum is detached in the exposed region and a the reduced E-cadherin signal indicates compromised cell junctions in the stratum basale (arrows). (d) A nevus (stained by hematoxylin and eosin) with tissue ablations corresponding to the exposure mask geometry and marked with black ink (arrows). Due the tenuity of the epidermis of only $40 \mu \mathrm{m}$, the damages reaches into the stratum basale. All scale bars correspond to $100 \mu \mathrm{m}$.

Figure 5 shows a few examples for these effects, all samples were exposed using a mask of ROIs at regular distances. The samples in Figure 5(a-c) were exposed to 100 $\mathrm{mW}$ laser power accoring to the protocol "Thermomechanical damage- full power". The sample in Figure 5(d) instead was exposed following "Thermomechanical damage- variable power". 
Figure 5(a) shows a sample of perilesional skin which manifests cavities in the stratum corneum having the same geometry as the exposure mask. The PAS staining highlights the intact basal membrane. Figure 5(b) shows the same sample immunostained with E-cadherin antibodies. The homogeneous expression in all epidermal layers proves the integrity of the extracellular matrix. Figure 5(c) shows an exposed basal-cell carcinoma which exhibits diminished E-cadherin expression in the superficial epidermal layers, indicating compromised intracellular junctions. The stratum corneum is detached and damaged. The only case when tissue ablation reached the basal cell layers is presented in Figure 5(d) showing a dermal nevus with an interrupted basal cell membrane in correspondence with the black ink that marks the exposed region.

\subsection{Power-compensated deep tissue imaging}

From these photodamage studies one can deduce depthdependent power limits, which allow the definition of safe power compensation protocols. While at a depth of $30 \mu \mathrm{m}$ for the described imaging parameters the average laser power should be limited to $20 \mathrm{~mW}$ to avoid structural damage, at $80 \mu \mathrm{m}$ the laser power limit can be relaxed to $50 \mathrm{~mW}$, and at $130 \mu \mathrm{m}$ even an increase to $80 \mathrm{~mW}$ will not cause any structural damage. These laser powers always refer to the values at the tissue surface.

Based on these threshold values an automatic in-depth power compensation scheme can be introduced into the image acquisition sequence. An optimum power compensation will increase the power exponentially to counteract the scattering losses of the excitation beam and the backtraveling fluorescence beam, both following LambertBeer's law. We found an increase of laser power from 3 $\mathrm{mW}$ for surface imaging to $30 \mathrm{~mW}$ for imaging at a depth of $200 \mu \mathrm{m}$, sufficient to compensate for the average loss of intensity.

As described in the methods section, experiments were performed to optimize the exponential power compensation constant $d$.
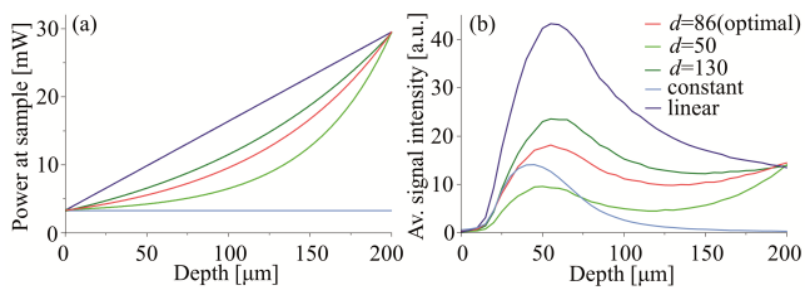

Figure 6 (a) Power compensation curves and (b) corresponding average fluorescence signals as a function of imaging depth. Different curves show different power compensation constants $d$ : constant power $(d=0)$ and $d=50 \mu \mathrm{m}$ were found to undercompensate scattering losses, $d=130 \mu \mathrm{m}$ and a linear function $(d>>1)$ overcompensate, while $d=86 \mu \mathrm{m}$ was found to give optimal results for this tissue type.
Different power compensation schemes were compared for $800 \mathrm{~nm}$ autofluorescence imaging (Figure 6(a)) starting from constant power and reaching via different exponential compensation constants $d$, to a linear compensation. The results (Figure 6(b)) show that uncompensated imaging leads to a complete loss of signal at $200 \mu \mathrm{m}$, while linear compensation causes a strong intensity overshoot in the region of maximum fluorescence at $50 \mu \mathrm{m}$. An exponential compensation with a constant $d=86 \mu \mathrm{m}$ was found to minimize the intensity variation as a function of imaging depth.

A volumetric reconstruction of a power-compensated optical biopsy is presented in Figure 7, where the grey scale represents the two-photon excited autofluorescence signal, the green signal shows the second harmonic generation from collagen fibres. A reasonable contrast can be maintained until an imaging depth of $200 \mu \mathrm{m}$.

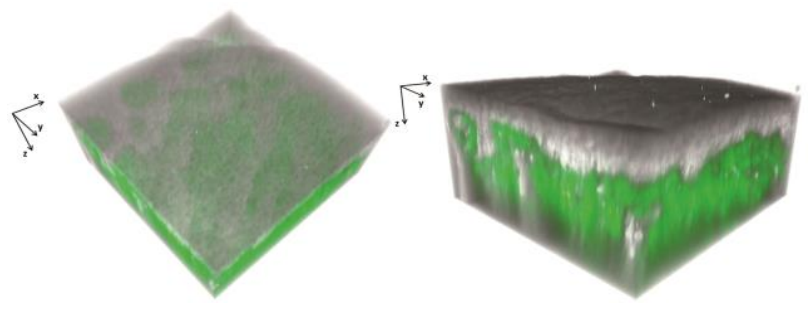

Figure 7 From a stack of 101 images at $2 \mu \mathrm{m}$ distance a 3D image was reconstructed via direct volume rendering. The cube dimensions are $352 \times 352 \times 200 \mu \mathrm{m}^{3}$. The grayscale shows the power-compensated autofluorescence signal, the green colour map reflects the second harmonic generation signal

\section{Discussion}

Within these studies excised human skin tissue samples were repeatedly exposed to fs-laser radiation as used in two-photon microscopy. Different damaging mechanisms were identified and characterized.

With increasing laser power, the first observed phenomenon was photobleaching. Two different processes were eminent: a fast one, power-independent, which was observed only in fast frame rate experiments. This indicates a reversible bleaching process with a recovery time so short, that the low frame rate exposure intervals of $1.7 \mathrm{~s}$ did not resolve it. Moreover, the lack of a power dependence may be explained by the fact that this process is already saturated at minimum applied laser power of $10 \mathrm{~mW}$ at the sample surface.

The second bleaching process was power-dependent and irreversible, signs of photochemical reactions (e.g. the formation of cytotoxic reactive oxygen species [23]). Significant permanent photobleaching was observed at laser powers above $10 \mathrm{~mW}$. The measured bleaching rates increased with increasing laser power, with a dependency 
that could be well characterized with power laws. The corresponding exponents varied in different tissue regions between 2 and 4 . This indicates the involvement of various nonlinear photobleaching pathways. However, the monitored dependence of the fluorescence intensity on the laser power was never higher than quadratic, so a direct absorption of three and more photons can be ruled out. The possible underlying processes are sketched in Figure 8.

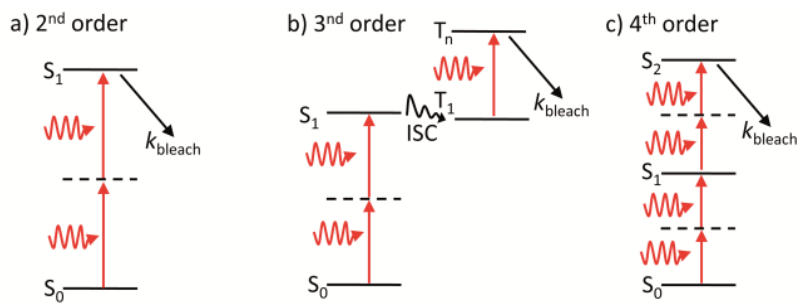

Figure 8 Photobleaching decay processes: after two-photon excitation (a); after two-photon excitation, intersystem crossing (ISC) and excitation into a higher triplet state (b); after double two-photon excitation to the second excited singlet state (c).

Besides bleaching from the two-photon excited singlet state showing quadratic intensity dependence of the decay rate (Figure 8(a)), a 3rd order power dependence could be explained by a two photon excitation followed by intersystem crossing and a subsequent single photon excitation to a higher order triplet state from which the molecule is bleached [24,25] (Figure 8(b)). Instead a 4th order dependence of the bleaching rate is most likely caused by two sequential biphotonic excitations into the second excited singlet state [22] (Figure 8(c)).

From in vitro studies one knows that photochemical reactions going along with photobleaching are causing functional damage, but since this study was performed on fixed tissue samples, there is no way to further quantify these consequences. In vitro, fs-radiation caused reduced cell reproducibility [12], evoked DNA strand breaks [19], and increased the creation of cyclobutane-pyrimidine-dimers in DNA [15]. An in vivo experiment showed enhanced lethality in Drosophila larvae [26].

In this study, above $20 \mathrm{~mW}$ in all tissues, although with different thresholds, a sudden onset of a strong luminescence signal was observed. The underlying damage process is most likely photoionization due to the high peak intensities at the focus $[9,12,22]$. A possible explanation for the signal is that free electrons are reducing $\mathrm{NAD}^{+}$to NADH [27]. Since the absorbance of NADH is much higher than of $\mathrm{NAD}^{+}$for two NIR photons, this reduction would strongly increase the autofluorescence signal. The effect sets in first in melanoma which could be due to the enhanced concentration of melanin. Melanin, being a strong absorber, would increase energy deposition in the sample, enhancing photoionization.
A following histological analysis did not show any structural change in tissues that exhibited photobleaching or luminescence increase. So these effects are important to be considered in evaluation of in vivo damage effects, the outcome of a subsequent histological analysis of fixed samples is unaffected.

At only slightly higher laser powers also structural damage effects started to occur. Cavities started to be formed in the tissue, which can be attributed to thermomechanical damage after single photon absorption in melanin causing tissue heating. The effect was limited to the exposed region. These structural damages were clearly visible also in the histological analysis of the exposed samples. The cavity pattern matches well the exposure masks apart from the fact that it is slightly above the 30 $\mu \mathrm{m}$ focal plane. This can be attributed to two effects: overall tissue shrinkage during tissue dehydration and embedding, and a shadow effect due to strong absorption which causes the laser intensity to be lower in layers slightly below the focus than above. Structural damage was usually limited to the stratum corneum. The only case were it reaches the basal layer may be due to the hypotrophy of the epidermis in an old patient (Figure 5(d)). In another case, and only beyond $80 \mathrm{~mW}$, the intracellular junctions in the stratum basale were found to be compromised showing reduced E-Cadherin levels. So generally the technique neither causes irreversible damage nor alters the histological diagnosis.

In all tissues structural damage threshold were beyond 35 $\mathrm{mW}$, agreeing with the pulse energies measured in [16], but interestingly, the luminescence increase, which always sets in at lower powers in our experiment, was not reported there.

The observed depth-dependence of all damaging effects permits to relax power limits in deeper layers, allowing two-photon optical biopsies with scattering-loss compensation down to $200 \mu \mathrm{m}$.

\section{Conclusion}

In this study different photodamage mechanisms induced by near-infrared femtosecond laser radiation in thick skin samples were identified and characterized regarding power- and imaging-depth-dependence. With increasing power, first photobleaching sets in. Its energy dependence suggests different involved bleaching pathways. At increased power a luminescence enhancement indicates photoionization. Both effects were limited to the stratum corneum and did not show any sign of tissue alteration in a histological analysis. Only at higher powers, thermomechanical cavitation caused structural damage. But these damages were focal within the superficial layers and a morphological damage of the basal layer was usually not observed. Experiments were performed on fixed tissue 
samples, which limited the analysis to structural changes. The observed power and depth-dependence of all effects allowed to establish a compensation protocol for damagefree optical biopsies with depth-dependent laser power adaptation. This reduces scattering losses in deep tissue imaging, allowing for a complementary analysis of skin biopsies with two-photon microscopy without altering the results of a subsequent histological analysis.

\section{Acknowledgement}

The authors acknowledge financial support by the Provincia autonoma di Trento project PAT Sanità 2009.

Author biographies Please see Supporting Information online.

\section{References}

[1] M. B. Lens and M. Dawes, Br. J. Dermatol. 150, 179 (2004).

[2] J. Ferlay, E. Steliarova-Foucher, J. Lortet-Tieulent, S. Rosso, J. W. W. Coebergh, H. Comber, D. Forman, and F. Bray, Eur. J. Cancer 49, 1374 (2013).

[3] M. Ulrich and S. Lange-Asschenfeldt, J. Biomed. Opt. 18, 061212 (2013).

[4] A. M. Zysk, F. T. Nguyen, A. L. Oldenburg, D. L. Marks, and S. A. Boppart, J. Biomed. Opt. 12, 051403 (2007).

[5] K. König, J. Biophotonics 1, 13 (2008).

[6] R. W. Boyd, Nonlinear Optics (San Diego, CA (United States); Academic Press Inc., 1992).

[7] W. R. Zipfel, R. M. Williams, and W. W. Webb, Nat. Biotechnol. 21, 1369 (2003).

[8] K. M. Hanson and C. J. Bardeen, Photochem. Photobiol. 85, 33 (2009).

[9] K. König, P. T. C. So, W. W. W. Mantulin, B. J. Tromberg, and E. Gratton, J. Microsc. 183, 197 (1996).

[10] O. Nadiarnykh, G. Thomas, J. Van Voskuilen, H. J. C. M. Sterenborg, and H. C. Gerritsen, J. Biomed. Opt. 17, 116024 (2012)
[11] Y. Sako and A. Sekihata, J. ... 185, 9 (1997).

[12] K. König, P. T. C. So, W. W. Mantulin, and E. Gratton, Opt. Lett. 22, 135 (1997).

[13] K. König, T. W. Becker, P. Fischer, I. Riemann, and K.-J. Halbhuber, Opt. Lett. 24, 113 (1999).

[14] A. Hopt and E. Neher, Biophys. J. 80, 2029 (2001).

[15] F. Fischer, B. Volkmer, S. Puschmann, R. Greinert, E. Breitbart, J. Kiefer, and R. Wepf, J. Biophotonics 1, 470 (2008).

[16] B. R. Masters, P. T. C. So, C. Buehler, N. Barry, J. D. Sutin, W. W. Mantulin, and E. Gratton, J. Biomed. Opt. 9, 1265 (2004).

[17] F. Fischer, B. Volkmer, S. Puschmann, R. Greinert, W. Breitbart, J. Kiefer, and R. Wepf, J. Biomed. Opt. 13, 041320 (2010).

[18] H. J. Koester, D. Baur, R. Uhl, and S. W. Hell, Biophys. J. 77, 2226 (1999).

[19] U. K. Tirlapur, K. König, C. Peuckert, R. Krieg, and K.-J. Halbhuber, Exp. Cell Res. 263, 88 (2001).

[20] G. Thomas, O. Nadiarnykh, J. van Voskuilen, C. L. Hoy, H. C. Gerritsen, and H. J. C. M. Sterenborg, J. Biophotonics 7, 492 (2014).

[21] S. L. Jacques and D. J. McAuliffe, Photochem. Photobiol. 53, 769 (1991).

[22] L. M. Tiede and M. G. Nichols, Photochem. Photobiol. 82, 656 (2006).

[23] A. Diaspro, G. Chirico, C. Usai, P. Ramoino, and J. Dobrucki, in Handb. Biol. Confocal Microsc., edited by J. B. Pawley, 3rd ed. (Springer, New York, 2006), pp. 690-702.

[24] E. J. Sánchez, L. Novotny, G. R. Holtom, and X. S. Xie, J. Phys. Chem. A 101, 7019 (1997).

[25] J. Widengren and R. Rigler, Bioimaging 4, 149 (1996).

[26] I. Saytashev, S. N. Arkhipov, N. Winkler, K. Zuraski, V. V Lozovoy, and M. Dantus, J. Photochem. Photobiol. B. 115, 42 (2012).

[27] D. Pestov, Y. Andegeko, V. Lozovoy, and M. Dantus, J. Opt. 084006, (2010). 